\title{
Tag location method integrating GNSS and RFID technology
}

\author{
Meng $\mathrm{Du}^{1,2}$, Changfeng Jing ${ }^{1}$ and Mingyi $\mathrm{Du}^{1^{*}}$
}

\begin{abstract}
Radio frequency identification (RFID) technology has become one of the most prosperous positioning technologies due to its advantages in non-contact and non-line-of-sight sensing. Most of the current positioning applications using RFID were implemented by setting a few RFID readers in some known locations, which were only suitable for the indoor or small area of outdoor positioning. This paper proposed a positioning algorithm by combining vehicle Global Navigation Satellite System (GNSS) and mobile RFID readers. The positioning accuracy is better than $\pm 5 \mathrm{~m}$ with an identification distance of $160 \mathrm{~m}$. This method can be used in the outdoor positioning with a rapid and accurate locating of the target objects, which is very helpful for the specific objects positioning and change detection in daily urban management and regulations.
\end{abstract}

Keywords: Tag position, GNSS, RFID

\section{Introduction}

According to Gartner Inc., location-awareness is one of the key technologies in the next few years (GARTNER 2012). Location services are traditional determined by using network and/or mobile-device-based technology (Schmidt-Dannert \& SNET 2011). Technologies include cell of origin (also known as cell ID), Angle of arrival (AOA), time of arrival (TOA) and Global Position System (GPS) or assisted GPS (Jing et al. 2015). Location services are widely used in urban management business. Many urban management components, such as breakfast cart, newsstands and billboards, are movable which often resulted in the negative influence on the traffic, cityscape and urban environment (Wu et al. 2008). How to location these movable urban management components rapidly and effectively has become an urgent issue for the urban management administrator.

Global Navigation Satellite System (GNSS) can be used outdoor without network modification but requires signal availability which is weak in urban complex environment. Therefore, the GNSS location methods can not enough for the dynamic location requirement of urban management business. With the rapid development of

\footnotetext{
* Correspondence: dumingyi@bucea.edu.cn

${ }^{1}$ School of Geomatics and Urban Information, Beijing University of Civil Engineering and Architecture, No. 1, Zhanlanguan Road, Xicheng District, Beijing 100044, China

Full list of author information is available at the end of the article
}

the Internet of Things (IOT), automated locating technologies such as Radio Frequency Identification (RFID) have proven to be beneficial in management and tracking urban infrastructures. For application research, RFID and ontology are used to location the roadside tree (Kikushige et al. 2011). RFID is used to vehicle location in complex environments such as tunnel and built-ups (Ning et al. 2013). Liu researched location application of urban signboard by RFID (Liu et al. 2012). Integrated method based on GPS and RFID is used to automate the identification and localization of construction components on large project, in which the GPS is for outdoor devices position and RFID is for indoor or lower signal (Torrent \& Caldas 2009). For location algorithm research, to overcome some uncertainty in RFID location, Hierarchical Algorithm by fusing an RFID system with an ultrasonic sensor system was proposed (Byoung-Suk et al. 2011). An improved GPS/RFID integration method based on Sequential Iterated Reduced Sigma Point Kalman Filter (SIR-SPKF) was proposed for vehicle navigation application (Peng et al. 2012). For the large shadowing fading problem in wireless indoor location, an indoor location algorithm based on maximum likelihood estimation was developed (Wei et al. 2006). Nearest neighbor algorithm based on reference tags is the most common used algorithm in RFID location ( $\mathrm{Ni}$ et al. 2003). Based on the historical track of object tags, a 


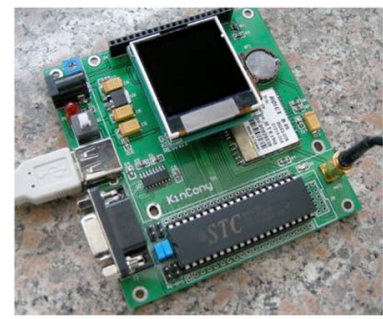

a

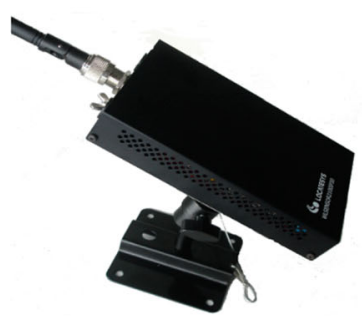

b

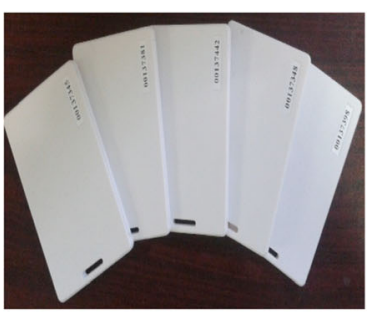

C

Fig. 1 Tag location device. a Vehicle mounted GNSS; b RFID reader; c RFID tags

method dynamic setting of $\mathrm{k}$ value was developed to improve nearest neighbor algorithm accuracy (Wang et al. 2010). Although many research for RFID location, the coordination of RFID was often jointed computed by fixed RFID network (components with RFID readers or tags) with known coordination (Ashok \& Marie 2013; Ning et al. 2013; Kuriakose et al. 2014) in above studies. In other words, a prepared network with reference tags or RFID readers must be used, which is cost and impractical for big city.

This paper proposed a novel tag positioning method based on Global Navigation Satellite System (GNSS) and RFID. The location device is composed of GNSS and RFID reader which are mounted on vehicle. The GNSS provides the vehicle position. The RFID reader can get the distance to target RFID tags. Based on the vehicle coordinate and distance, the target tags can be located. This paper is organized as follow. In Tag location method based on GNSS and RFID section, we provide the detail design and implementation of proposed method. Experiments section presents the experiment and data analysis. Conclusion section draws conclusion and gives suggestion for future work.

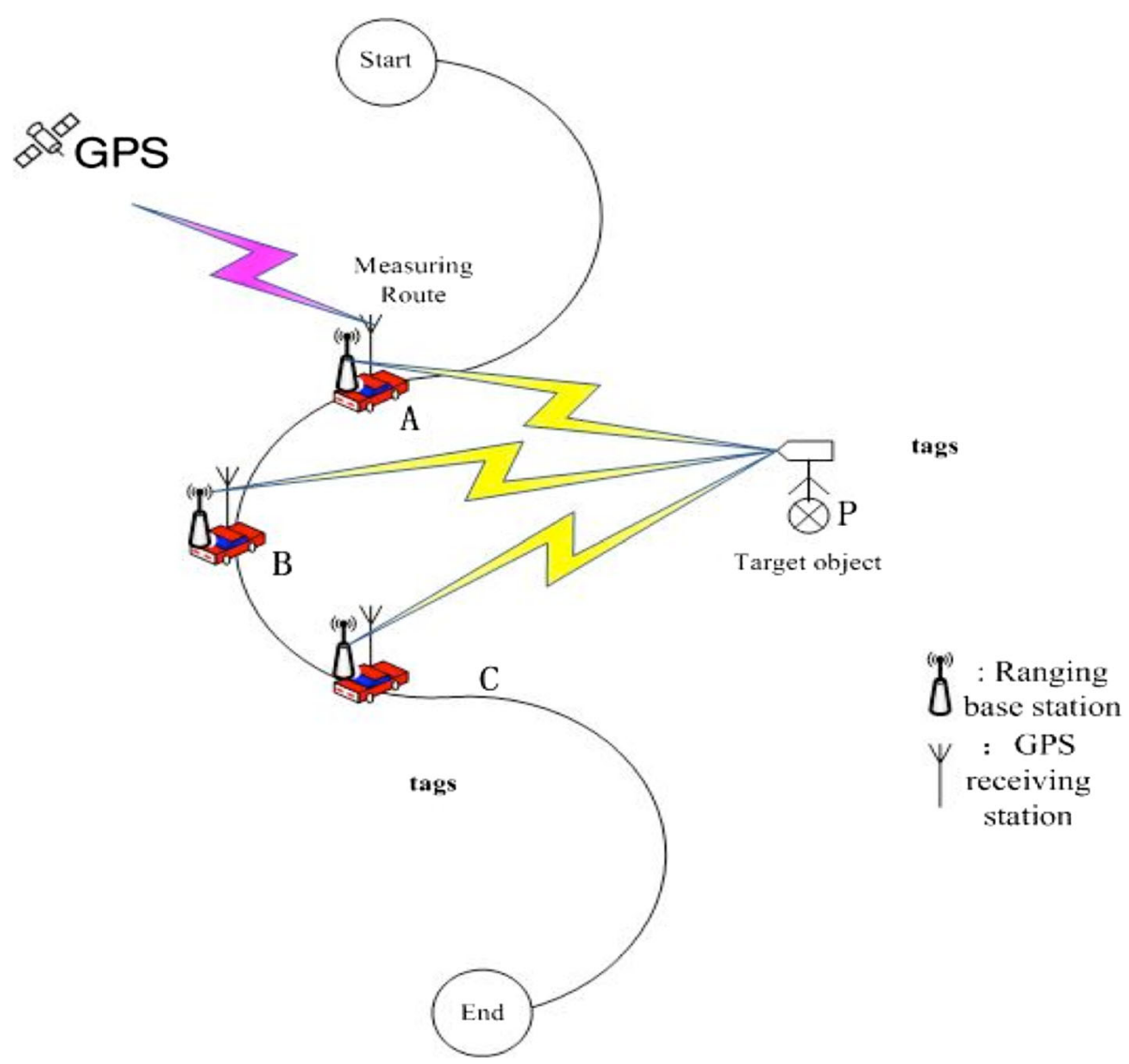

Fig. 2 Triangulation principle 


\section{Method}

\section{Components for positioning system}

This device consists of vehicle GNSS module and RFID module as shown in Fig. 1. The former is responsible for the real-time positioning of the RFID reader. It includes GNSS receiving antenna, GNSS chips, GNSS software shown in Fig. 1a. The later module is employed to capture the signals of RFID reader (shown in Fig. 1b) and RFID tags (shown in Fig. 1c) and then to calculate distance.

\section{Location algorithm}

\section{Workflow of location algorithm}

The principle of proposed algorithm is simulating the radio frequency base station network by rapid movable RFID readers. An RFID tag is equipped on the target points. The RFID reader continues receiving the tag's signal when the vehicle is moving within the ranging distance, so that distance between RFID reader and tag can be calculated. The location of RFID reader is gotten by the vehicle GNSS. Based on distance and RFID reader coordinate the target tags can be located. This principle is based on the theory of "triangulation" showed in Fig. 2, where $A, B$ and $C$ are three different points for the movable RFID readers respectively, called ranging points; $P$ is the target object which equipped with target tag, called target tag. Figure 3 shows the detailed workflow of algorithm.

The detailed workflow of algorithm includes follow five steps.

i). Data capture including both GNSS and RFID data when vehicle is moving.

ii). Data optimization includes filtering bad ranging points data and GNSS data.

iii). Data synchronization for ranging points and GNSS points based on time.

iv). Data classification which means the data with specific ID is assigned to the corresponding target tag;

v). Target tag' coordinate calculation based on more than 3 ranging points.

\section{Data optimization}

In position, some range discontinuity point caused by RFID reader low voltage or some occludes must be removed. The filtered points are used to compose the triangles with target tags according to triangulation, as shown in Fig. 4. When the vehicle is moving, more range points are generated. Every two continuous points jointing one target tag forms a triangle. While vertex angle is less than $15^{\circ}$, this triangle is regarded as ill-conditioned triangle and removed from the calculation. In Fig. $4 \mathrm{a}$, the angle $\angle B P_{3} C$ is less than $15^{\circ}$,

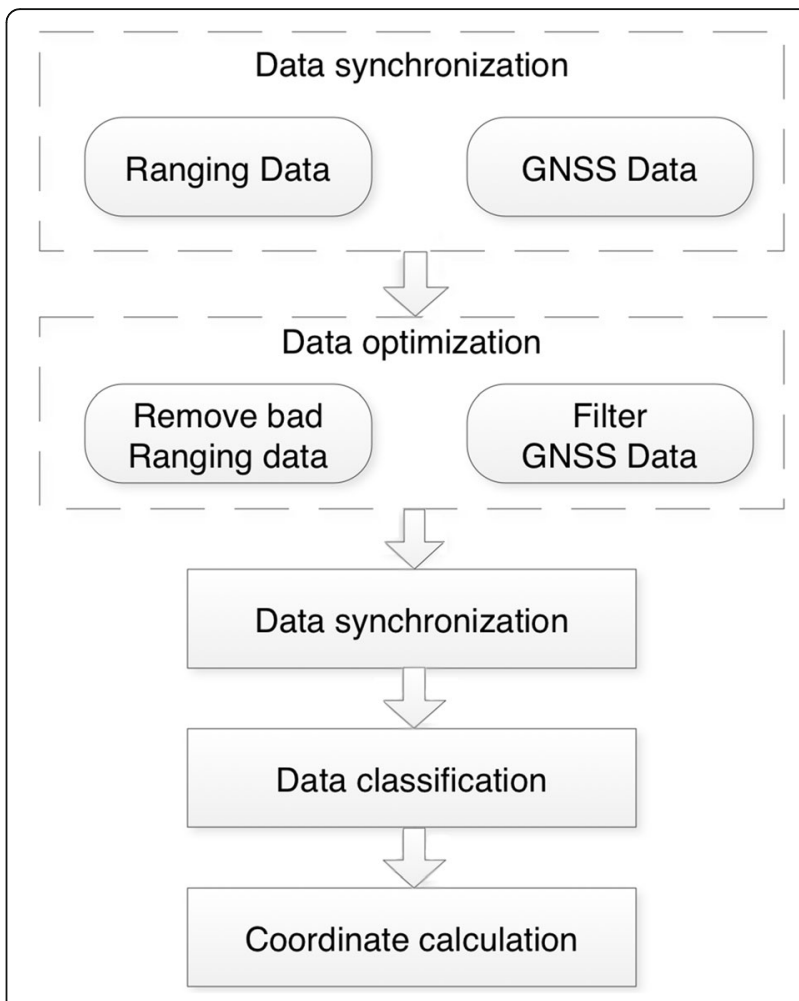

Fig. 3 Workflow of algorithm

therefore triangle $B P_{2} P_{3}$ is to be removed. With this step, only the good ranging points are saved.

\section{Coordinate calculation}

As shown in Fig. 4b, the coordinate values of points $A$, $B, C$ are gotten by vehicle mounted GNSS devices. The distance between two points is calculated, and then the position of target point $P$ can be calculated by equation (1) according to triangulation.

$$
\left\{\begin{array}{l}
\left(x_{p}-x_{a}\right)^{2}+\left(y_{p}-y_{a}\right)^{2}=d_{a}^{2} \\
\left(x_{p}-x_{b}\right)^{2}+\left(y_{p}-y_{b}\right)^{2}=d_{b}^{2}
\end{array}\right.
$$

Where, $A\left(x_{a}, y_{a}\right)$ and $B\left(x_{b}, y_{b}\right)$ are two known points, the distance between $A$ and $P$ is $d_{a}$, and $d_{b}$ is the distance between $B$ and $P$, and then the $P\left(x_{p}, y_{p}\right)$ is the calculated coordinate. But, based on theory of triangle geometry, there are two triangles if known all three sides of a triangle. It is means there are two possible position of target point $P$ (point $P$ and $P_{1}$, as shown in Fig. $4 \mathrm{~b}$ ), which are lies two side of line $A$ and $B$. From the algebra, equation (1) is a binary quadratic polynomial, and may be has 0,1 or 2 possible solutions. Integrating the triangle geometry, in this paper, it has two solutions. To determine the right position for target tag, three or more ranging points are included in calculation. Each two of 


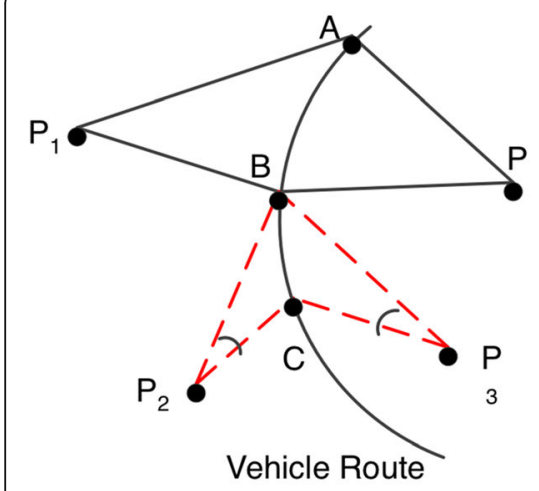

a

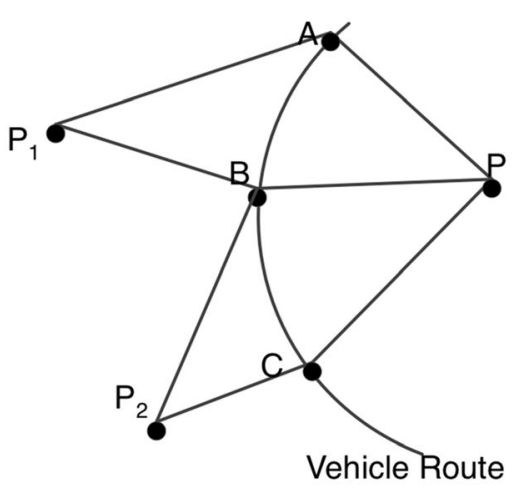

b

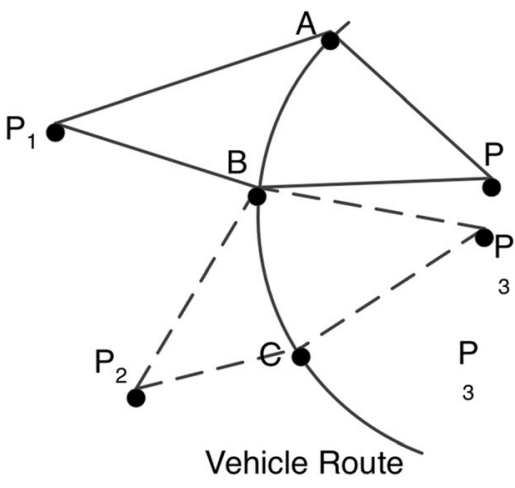

C

Fig. 4 Proposed location algorithm. a ill-conditioned triangle; $\mathbf{b}$ theory model of triangulation; $\mathbf{c}$ practical model of triangulation. Where, $A, B, C$ are ranging point; $P, P_{1}, P_{2}, P_{3}$ are target tags; the arc represents the vehicle route

these ranging points jointed target point can form a triangle and get two possible target tag positions. But one of two positions is the same to all possible target tags. For example in Fig. 4b, ranging points $A$ and $B$ can form a triangle with target tag, and get two possible position $P$ and $P 1$. Ranging point $B$ and $C$ can form another triangle and get two candidate positions $P$ and $P 2$. Point $P$ is the common point in two triangles; therefore $P$ is the right target tag position.

However, above is the theory model. In practice, the possible position cannot be same to next solution due to ranging error. The true scene may like Fig. 4c. The two possible target tag position is near. To determine the right position, only two points with smallest distance are used to calculate, which means the higher accuracy. As shown in Fig. 4c, the point $P$ and $P_{3}$ are candidate position. The simple arithmetic mean algorithm is adopted to determine the final position. If the coordinate for $\mathrm{P}$ is $\left(x_{p}, y_{p}\right)$, and $\mathrm{P}_{3}$ is $\left(x_{p 3}, y_{p 3}\right)$, the right coordinate of target tag can be calculated by equation (2).

$$
\left\{\begin{array}{l}
x_{p o}=\frac{x_{p}+x_{p 3}}{2} \\
y_{p o}=\frac{y_{p}+y_{p 3}}{2}
\end{array}\right.
$$

\section{Results}

The experiment is designed and executed with the device as Components for positioning system section.

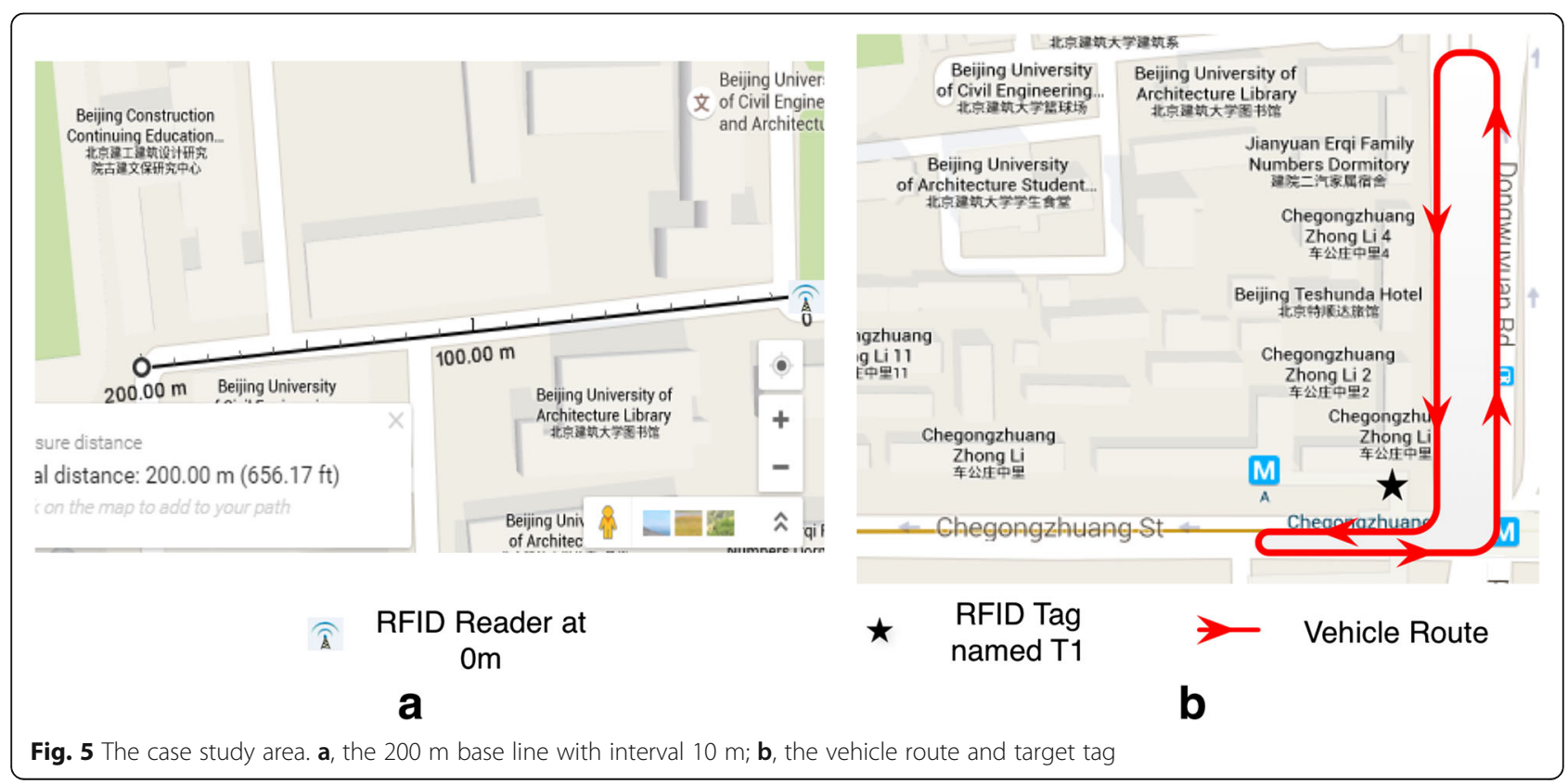


Table 1 Ranging experiment data

\begin{tabular}{|c|c|c|c|c|c|}
\hline Preset distance (m) & Measured distance $(\mathrm{m})$ & Difference $(\Delta)$ & Preset distance $(\mathrm{m})$ & Measured distance $(\mathrm{m})$ & Difference $(\Delta)$ \\
\hline 10 & 10.13 & +0.13 & 110 & 107.49 & -2.51 \\
\hline 20 & 19.93 & -0.07 & 120 & 119.10 & -0.80 \\
\hline 30 & 29.90 & -0.10 & 130 & 127.23 & -2.77 \\
\hline 40 & 38.89 & -1.11 & 140 & 142.07 & +2.07 \\
\hline 50 & 51.32 & +1.32 & 150 & 152.93 & +2.93 \\
\hline 60 & 60.99 & +0.99 & 160 & 160.54 & +0.54 \\
\hline 70 & 72.10 & +2.10 & 170 & 174.10 & +4.10 \\
\hline 80 & 78.73 & -1.27 & 180 & 185.58 & +5.58 \\
\hline 90 & 88.32 & -1.68 & 190 & 194.38 & -4.38 \\
\hline 100 & 102.24 & +2.24 & 200 & 203.65 & +3.65 \\
\hline
\end{tabular}

The case study area lies in Xicheng district, Beijing, China. The road, where is the main road of local area shown in Fig. 5, is selected as vehicle route. A vehicle mounted GNSS (GPS) receiver moved along the vehicle route. The purpose of experiment is to check the ranging data and tag position accuracy.

For ranging data verification, a $200 \mathrm{~m}$ base line composed of 20 points with interval $10 \mathrm{~m}$ is designed, as shown in Fig. $5 \mathrm{a}$. The true value of 20 points is gotten by Leica GPS (a high accurate survey device). The accuracy of ranging data is compared to its' true value. Therefore, the threshold ranging data is validated for higher accuracy.

For tag position accuracy verification, several fixed points with true value are selected and deployed a tag respectively, shown in Fig. 5b. As the vehicle moving along route, target tag coordinate are calculated. Comparing the measured tag position and true value of points, the accuracy is validated.

Table 2 Tag positioning data and error

\begin{tabular}{|c|c|c|c|c|c|c|c|}
\hline \multirow[t]{2}{*}{ No } & \multirow{2}{*}{$\begin{array}{l}\text { Total } \\
\text { triangles }\end{array}$} & \multirow{2}{*}{$\begin{array}{l}\text { Ill-conditioned } \\
\text { triangles }\end{array}$} & \multicolumn{2}{|c|}{ Calculated coordinate value } & \multicolumn{2}{|c|}{ Difference } & \multirow{2}{*}{$\begin{array}{l}\text { Closing } \\
\text { error }(\mathrm{m})\end{array}$} \\
\hline & & & $(X)$ & $(Y)$ & $(\Delta X)$ & $(\Delta Y)$ & \\
\hline 1 & 1274 & 418 & 498846.6 & 307259.0 & -0.906 & 3.375 & 3.49569 \\
\hline 2 & 2653 & 611 & 498849.2 & 307257.6 & 1.718 & 2.001 & 2.63706 \\
\hline 3 & 1895 & 503 & 498849.1 & 307257.7 & 1.625 & 2.062 & 2.62574 \\
\hline 4 & 968 & 285 & 498847.0 & 307258.7 & -0.503 & 3.125 & 3.15918 \\
\hline 5 & 2003 & 583 & 498846.6 & 307259.0 & -0.906 & 3.375 & 3.49455 \\
\hline 6 & 1632 & 455 & 498849.2 & 307257.6 & 1.718 & 2.004 & 2.63706 \\
\hline 7 & 3502 & 1303 & 498849.1 & 307257.7 & 1.625 & 2.062 & 2.62574 \\
\hline 8 & 2838 & 1032 & 498847.0 & 307258.7 & -0.501 & 3.125 & 3.15981 \\
\hline 9 & 1697 & 477 & 498848.5 & 307254.5 & 1.014 & -1.093 & 1.48198 \\
\hline 10 & 1435 & 303 & 498854.7 & 307256.9 & 7.218 & 1.281 & 7.33157 \\
\hline 11 & 2114 & 689 & 498851.6 & 307256.2 & 4.093 & 0.562 & 4.13222 \\
\hline 12 & 3026 & 827 & 498848.9 & 307254.8 & 1.406 & -0.812 & 1.62409 \\
\hline 13 & 993 & 266 & 498851.3 & 307256.2 & 3.843 & 0.593 & 4.26685 \\
\hline 14 & 1064 & 253 & 498849.7 & 307259.2 & 2.254 & 3.625 & 4.26658 \\
\hline 15 & 2866 & 764 & 498848.2 & 307254.5 & 0.751 & -1.061 & 1.30054 \\
\hline 16 & 2735 & 930 & 498848.7 & 307254.8 & 1.254 & -0.781 & 1.47472 \\
\hline 17 & 1343 & 444 & 498850.1 & 307255.3 & 2.593 & -0.312 & 2.61288 \\
\hline 18 & 1204 & 373 & 498847.8 & 307254.3 & 0.375 & -1.281 & 1.33528 \\
\hline 19 & 862 & 265 & 498849.1 & 307255.0 & 1.656 & -0.625 & 1.76678 \\
\hline 20 & 2966 & 1147 & 498851.4 & 307255.4 & 3.937 & -0.156 & 3.94059 \\
\hline
\end{tabular}




\section{Discussion}

Followed above experiment design, 20 group data with intervals of $10 \mathrm{~m}$ are collected in the case study area, as shown in Table 1 . The preset distance represents the true value distance from the RFID reader to check point of base line, which is gotten by Leica GPS device. Measured distance is the ranging distance between reader and target tag. Difference shows the absolute error of ranging data. The smaller of absolute error means the higher ranging data accuracy. As shown in Table 1, the difference is less than 3.0 when the distance is smaller than $160 \mathrm{~m}$. Otherwise, the difference is significantly increased. Therefore, the $160 \mathrm{~m}$ is selected the threshold of higher ranging data under multiple experiment test.

Followed the experiment, the 20 group tag positioning data of tag $T 1(498847.463,307255.556)$ is gotten under the $160 \mathrm{~m}$ ranging threshold value, shown in Table 2. Illconditioned triangles were removed in calculation. The column "difference" means the absolute error of $\mathrm{X}$ and $\mathrm{Y}$ coordinates, and the column "closing error" is the target tag position absolute error. Mostly absolute error falls in $[-5 \mathrm{~m},+5 \mathrm{~m}]$. Only one big closing error is $+7.33 \mathrm{~m}$, which is caused by fading of moving bus in line-of-sight.

\section{Conclusion}

The coordinates of the movable tags are calculated based on many position fixed radio frequency RFID readers in the traditional radio frequency positioning method. This paper proposed an algorithm by using a single movable radio frequency RFID reader and vehicle GNSS to compute the position of static tags. A vehicle inspection and supervision system has been developed based on this algorithm. The positioning accuracy achieved in this system is better than $5 \mathrm{~m}$ within a distance of $160 \mathrm{~m}$, such that this system is good enough for the management of the breakfast vendors, newsstands, billboards and other urban management case studies, which provides a new convenient and accurate approach for the urban supervision and management.

\section{Acknowledgement}

The authors would like to express appreciations to colleagues in our laboratory for their valuable comments and other helps. This research is partially supported by Beijing Natural Science Foundation (Series B) granted No.KZ201210016016, Key Laboratory for Urban Geomatics of National Administration of Surveying, Mapping and Geoinformation granted NO.20141204NY and Science Foundation of Beijing University of Civil Engineering and Architecture granted NO.ZF15071.

\section{Competing interests}

All authors have seen the manuscript and approved to submit to this journal. The authors declare that they have no competing interests.

\section{Authors' contributions}

$M D, C J$ and $M D$ conceived and designed the study. MD and CJ developed the system and process the figures. MD contributed in the preparation of the manuscript. All authors read and approved the manuscript.

\section{Author details}

${ }^{1}$ School of Geomatics and Urban Information, Beijing University of Civil Engineering and Architecture, No. 1, Zhanlanguan Road, Xicheng District, Beijing 100044, China. ${ }^{2}$ Beijing Intel-city Spatial Information Technology Co. Ltd, No. 46, Jiaodadong Road, Haidian District, Beijing 100044, China.

Received: 16 July 2016 Accepted: 18 July 2016

Published online: 04 November 2016

\section{References}

Ashok P, Marie KB (2013) A Survey of Positioning Algorithms on Mobile Devices in Location Based Services. Int J Adv Res Comput Sci Softw Eng 3(6):1778-1784

Byoung-Suk C, Lee JW, Ju-Jang L, Kyoung-Taik P (2011) A Hierarchical Algorithm for Indoor Mobile Robot Localization Using RFID Sensor Fusion. IEEE Trans Ind Electron 58(6):2226-2235

GARTNER, Inc (2012) Gartner Highlights Top Consumer Mobile Applications and Services for Digital Marketing Leaders. http://www.gartner.com/newsroom/ id/2194115. Accessed 6 Nov 2016.

Jing C, Du M, Shang L (2015) A low-cost RFID/GPS location sensing algorithm for urban infrastructure. Int J Online Eng 11(4):4-7

Kikushige Y, Yabuki N, Fukuda T (2011). A Management System of Roadside Trees Using RFID and Ontology. In: Computing in Civil Engineering 2011.

American Society of Civil Engineers, Reston, Virginia, 2011, pp 307-314.

Kuriakose J, Joshi S, Raju RV, Kilaru A (2014) A review on localization in wireless sensor networks. Advances in signal processing and intelligent recognition systems. Springer, In, pp 599-610

Liu Y, Du M, Jing C, Shi R (2012) Vehicle-mounted outdoor signboard management system combinging MMS and RFID technologies. In: International Geoscience and Remote Sensing Symposium (IGARSS) 2012. Institute of Electrical and Electronics Engineers Inc., Munich, Germany, pp 2763-2766

Ni LM, Yunhao L, Yiu CL, Patil AP (2003) LANDMARC: indoor location sensing using active RFID. In: In: Proceedings of the First IEEE International Conference on Pervasive Computing and Communications, 2003. (PerCom 2003)., pp 407-415

Ning Y, Zhong-qin W, Malekian R, Ru-chuan W, Abdullah AH (2013) Design of Accurate Vehicle Location System Using RFID. ELEKTRONIKA IR ELEKTROTECHNIKA 19(8):105-110

Peng J, Wu FL, Zhu M, Wang FX, Zhang KF (2012) An Improved GPS/RFID Integration Method Based on Sequential Iterated Reduced Sigma Point Kalman Filter. leice Trans Commun E95B(7):2433-2441

Schmidt-Dannert A, SNET SMM (2011) Positioning Technologies and Mechanisms for mobile Devices. In: TU-Berlin (ed) Seminar Master Module SNET2

Torrent D, Caldas C (2009) Methodology for Automating the Identification and Localization of Construction Components on Industrial Projects. J Comput Civil Eng 23(1):3-13

Wang YZ, et al (2010) Research and application of RFID location algorithm based on reference tags. J Commun. 31(02):86-92. (In Chinese)

Wei N, Gang S, Xiaobing L, Luoning G (2006) An Indoor Location Algorithm Based on Taylor Series Expansion and Maximum Likelihood Estimation. In: Personal, Indoor and Mobile Radio Communications, 2006 IEEE 17th International Symposium on, in Editor (Neuvo, Yrjö), Helsiniki, Finland, 2006, pp 1-4.

WU C et al (2008) The Specific Application of Interest Point in the Urban Management Information System. Urban Manage Sci Technol 10(2):52-54, in Chinese

\section{Submit your manuscript to a SpringerOpen ${ }^{\circ}$ journal and benefit from:}

- Convenient online submission

- Rigorous peer review

- Immediate publication on acceptance

- Open access: articles freely available online

- High visibility within the field

- Retaining the copyright to your article

Submit your next manuscript at springeropen.com 